\title{
Inhibition effect of green alga on cyanobacteria by the interspecies interactions
}

\author{
J. Q. Chen • R. X. Guo
}

Received: 25 June 2012/Revised: 24 December 2012/ Accepted: 12 February 2013/Published online: 19 March 2013

(C) Islamic Azad University (IAU) 2013

\begin{abstract}
In the present study, we selected the common freshwater green alga Scenedesmus obliquus as the candidate cyanobacteria-control species by its allelopathic effect. The most common cyanobacterial species Microcystis aeruginosa was used as the target organism. The population growth of $M$. aeruginosa was inhibited by the green alga-conditioned media significantly. The influence was stronger when media concentrations increased from 0 to $100 \%$. In contrast, there was a strong and significant positive effect of the culture media from M. aeruginosa on the growth of green alga. Our results are useful and valuable as the experimental evidence for producing new cyanobacterial inhibitors and applying new environmentalfriendly biological control methods in future. For one thing, we could use the green alga-condition media but not the green alga itself to inhibit the growth of M. aeruginosa. For another, although cyanobacteria are harmful for the environment, the condition media from cyanobacteria could also be viewed as the resource to stimulate the growth of $S$. obliquus, which is advantaged for gaining more green alga-condition media and food for fish.
\end{abstract}

Keywords Allelopathic effect - Cyanobacterial control . Microcystis aeruginosa $\cdot$ Scenedesmus obliquus

Electronic supplementary material The online version of this article (doi:10.1007/s13762-013-0208-1) contains supplementary material, which is available to authorized users.

J. Q. Chen · R. X. Guo

Department of Environmental Science,

China Pharmaceutical University, 210009 Nanjing, China

J. Q. Chen $(\square)$

Department of Analytical Chemistry,

China Pharmaceutical University, 210009 Nanjing, China

e-mail: cjqalga@163.com

\section{Introduction}

Cyanobacterial blooms seem to be increasing in the shallow lakes world widely due to eutrophication (Collins 1978). These blooms have severe effects on the quality of water resources for drinking and agriculture, which can be harmful to both human and animal health (Collins 1978). Among the cyanobacterial species, Microcystis aeruginosa is one of the most common species and have caused disruptions of aquatic ecosystems (Zhang et al. 2009). Various strategies for the control of cyanobacterial blooms have been proposed over the last decades (Paerl et al. 2001), including physical measures, chemical usage, and biological control. However, physical methods have been application limited due to the high cost (Wu et al. 2010), while chemical usages are often associated with changes in $\mathrm{pH}$ values or salinity, which may threaten life in the lake (Hullebusch et al. 2002).

Biological control is an attractive option. In this strategy, the food chain and inhabitancy are reconstructed within the hydro-ecosystem and the biodiversity is recovered by bioand microbial-manipulations (Mehner et al. 2004). Although biological control processes may influence the dynamics of harmful blooms strongly (Mazzillo et al. 2011), they also lead other ecological risks. For example, although the cyanobacterial blooms could be controlled by zooplankton grazing, they also have the complex interactions with zooplankton, which could affect the grazing and reproduction of zooplankton (Cohen et al. 2007). The observation that cyanobacteria appear to be particularly susceptible to the active agent released by the barley straw offers a possible method of cyanobacteria control (Barrett et al. 1996). The indirect inhibition between interspecies via allelopathic compounds may have more significant influences on the phytoplankton activities (Fistarol et al. 2003). Green algae are one of the important components of the primary production in 
freshwater and the main food for fish (Lurling 2003). Thus, using the allelopathic effect from green algae species could be a possible new method to control cyanobacterial blooms. This research presented the laboratory experiments performed in culture flasks with the aim to observe the inhibition effect. We used the common freshwater green alga Scenedesmus obliquus as the candidate cyanobacteria-control species by its possible allelopathic effect. The whole experiments were carried out from 3 March 2010 to 1 June 2012 in the lab of Department of Environmental Science, China Pharmaceutical University, Nanjing, China.

\section{Materials and methods}

Test organisms and culture condition

Strains of the cyanobacteria M. aeruginosa (FACHB-1005) and the green alga $S$. obliquus (FACHB-416) were obtained from the Institute of Hydrobiology of the Chinese Academy of Sciences and grown as single cell in batch culture in mixed media (contain the basic ingredient including BG-11 medium and SE medium) in pre-culture time. Culture media were adjusted to pH 8.0 with $1 \mathrm{~mol} \mathrm{~L}^{-1} \mathrm{NaOH}$ and HCL. The cyanobacteria and the green alga were incubated and maintained at $26 \pm 1{ }^{\circ} \mathrm{C}$ under an illumination intensity of 2,000 lux, with a 12/12 h light/dark interval.

\section{Experiment design}

All the experiments were performed in two parts. First, we tested the possible inhibition effect of the green alga-conditioned media on the growth of cyanobacteria (Part I). M. aeruginosa were cultivated for 1 week before experiment. An inoculum was prepared for each experiment from fresh culture stocks sampled during the exponential growth phase. The cultivated cells were inoculated into the culture vessels at densities of $1 \times 10^{6}$ cell $\mathrm{mL}^{-1}$. The green alga-conditioned media used in the study were prepared from unispecies culture of S. obliquus before experiment. To avoid the nutrient level or starvation effect, the green alga was cultured in SE media with nitrogen and phosphorus in normal concentrations. After growing to the maximum density, the green alga with the conditioned media was $0.45 \mu \mathrm{m}$ filtered (Millex-GP, Millipore Co.) and the appropriate volumes of BG-11 media were added to each group providing the nominal concentrations in 5, 10, 20, 50, and $100 \%$. In Part II, we tested how the cyanobacteria-conditioned media affect the growth of $S$. obliquus. All the steps were as the same treatments as those in part I, while M. aeruginosa was cultured in BG-11 media in unispecies culture before experiment and the appropriate volumes of SE media were added to each group to provide the nominal concentrations in $5,10,20,50$, and $100 \%$.
Conditioned-free media was used as the control. Samples in any part were removed from the culture vessels at the predetermined times every day. Cells in the fixed samples were counted under microscope (BM-1000, China). The population growth rate $(r)$ was calculated from the formula: $r=1 /$ $t\left(\ln N_{t}-\ln N_{0}\right)$; where $N_{t}$ and $N_{0}$ are population sizes at day $0-t$, and $t$ is the time in days when the population size is maximum. Each experiment had three replications per treatment. All the experimental treatments were run under the same light and temperature conditions as the pre-culture conditions.

\section{Statistical analyses}

All the data analyses were carried out with the SPSS analytic package 16.0. Data were first tested for homogeneity (Levene's test). Results in the treatment groups were compared to controls by one-way analysis of variance (ANOVA) to identify the significant differences. Result were considered significantly different when $p<0.05$. All the figures were produced using Sigmaplot Version 11.0.

\section{Results and discussion}

The population growth curves of the cyanobacteria M. aeruginosa under the green alga-conditioned media in varied concentrations are presented in Fig. 1a. When M. aeruginosa was cultured under green alga-conditioned media in five concentrations, the population densities were significantly lower than that of the control. Although the population increased during the whole experiment time under any given concentration, the maximum population sizes were $23.50 \pm 0.50,21.25 \pm 1.25,14.25 \pm 1.75,14.23 \pm 0.75$, and $8.75 \pm 1.25 \times 10^{6}$ cell $\mathrm{mL}^{-1}$, respectively, which were only $74.60,67.46,45.24,45.17$, and $27.78 \%$ of that of the control $\left(31.50 \pm 5.50 \times 10^{6}\right)$. The inhibition effect of the green alga-conditioned media was significantly correlated.

In contrast, the population growth curve of the green alga $S$. obliquus under the cyanobacteria-conditioned media in varied concentrations during 12 days are presented in Fig. $1 \mathrm{~b}$. In general, the population under any concentration of the conditioned media increased during 12 days. The green alga population grew slowly in the first 4 days but then increased faster until the end of the experiment. When S. obliquus was cultured under the cyanobacteria-conditioned media in five concentrations $(5,10,20,50$, and $100 \%)$, the population densities were significantly higher than that of the control (Fig. 1b). The maximum population sizes were $5.30 \pm 0.50$, $5.65 \pm 0.35,9.60 \pm 0.60,10.50 \pm 0.50$, and $8.55 \pm 0.25 \times$ $10^{6}$ cell $\mathrm{mL}^{-1}$, respectively, which were $23.26,31.40$, 123.26, 144.19, and $98.84 \%$ more than that of the control $\left(4.30 \pm 0.10 \times 10^{6}\right)$. The trend in the influence of cyanobacteria-conditioned media was significantly correlated. 

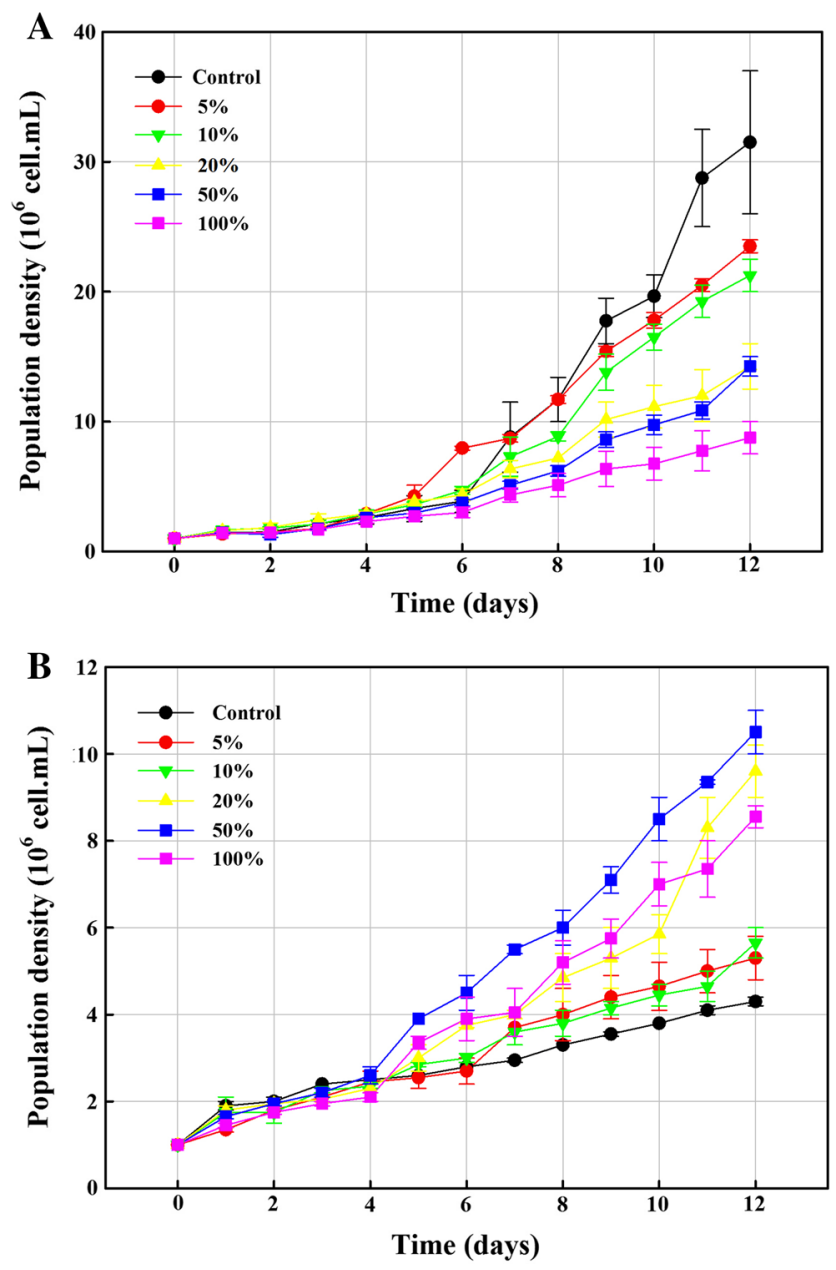

Fig. 1 Population growth curve of the cyanobacteria and green alga under the conditioned media in varied concentrations filtered through $0.45 \mu \mathrm{m}$ membrane during 12 days. a Microcystis aeruginosa cultured under green alga-conditioned media, b Scenedesmus obliquus cultured under cyanobacteria conditioned media

The population growth rates in varied concentrations media filtered through $0.45 \mu \mathrm{m}$ membrane are presented in Fig. 2, respectively. Under filtering treatment, when concentrations increased from 0 to $100 \%$, the population growth rates of $M$. aeruginosa declined from $0.29 \pm 0.01$ to $0.18 \pm 0.01$ (day $^{-1}$ ) (Fig. 2, blue points). Statistical analysis showed that the filtered media concentration had significant effect on the population growth $\left(F_{5,17}=50.37\right.$, $p<0.01, F$ test). The concentration-response relationship was observed clearly: the regression equation of population growth rate and filtered media concentration was linear $\left(y=0.31-0.02 x, R^{2}=0.95\right)$. In contrast, when the concentrations increased from 0 to $100 \%$, the population growth rate of $S$. obliquus increased from $0.12 \pm 0.002$ to $0.18 \pm 0.002\left(\right.$ day $\left.^{-1}\right)$ (Fig. 2, red points). Statistical analysis showed that the filtered media concentration had significant effect on the population growth $\left(F_{5,17}=117.40\right.$, $p<0.01, F$ test). The regression equation of the population

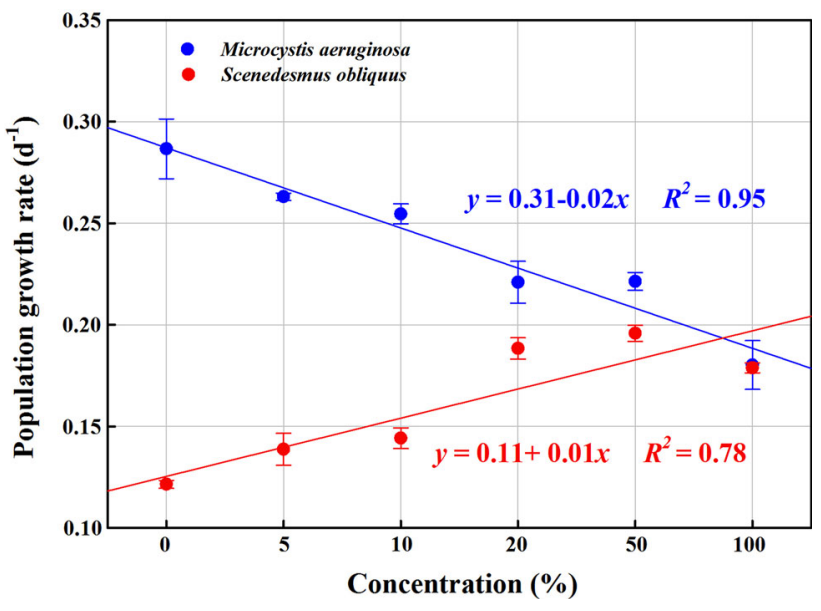

Fig. 2 Population growth rate of Microcystis aeruginosa and Scenedesmus obliquus in varied concentrations media filtered through $0.45 \mu \mathrm{m}$ membrane

growth rate and the filtered media concentration was linear $\left(y=0.11+0.01 x, R^{2}=0.78\right)$.

Allelopathic effects are characterized by the release of allelopathic compounds (allelochemicals) into the surrounding medium, eliciting either a positive or deleterious response in the target organism (Mulderij et al. 2005). In aquatic ecosystems, allelopathic effect is regarded as an important process influencing the shaping of microbial communities (Gross 2003; Hay 2009). Recent research showed that the main factor controlling allelochemical activity was the growth stage of the alga (Leãoa et al. 2010). Direct cell-cell interactions offer a possible transmission mechanism for allelopathic compounds (Jonsson et al. 2009). Dinoflagellate compound karlotoxin exerts its effect after cell encounters, suggesting this mechanism (Adolf et al. 2007). The important aspect of cell-cell interaction is that the allelopathic effect depends on the local concentration of a compound during encounters between susceptible and the allelopathic cells. Compared with the results, our experiments were performed using single species conditioned media from cultures of possible allelopathic species without cell-cell interaction driving, which could discriminate the indirect chemically mediated effects like allelochemicals from the direct contact. The population growth of the cyanobacteria $M$. aeruginosa was inhibited significantly by the chemical substances, which produced by the green alga $S$. obliquus itself in the preculture phase. The influence was stronger within the concentrations increased from 0 to $100 \%$ (concentration-response relationship). These results indicated that the chemical substances with allelopathic effect could occur at pre-culture conditions without two species cell-cell interaction. It has been proposed that the possible allelopathy might be explained a competitive advantage among cyanobacteria and green alga by their own chemical information naturally without interaction inducing. In 
contrast, there was a strong and significant positive effect of culture media from M. aeruginosa on the growth of green alga. Although similar results have not reported before and some biologists consider that only direct negative biochemical interaction should be included in allelopathy (Legrand et al. 2003), our study indicated that this definition should include not only inhibitory but also stimulatory effects.

Although the indirect chemical communication is a wellknown ecological phenomenon mediating interactions between zooplankton via conditioned media without encounter (Guo et al. 2011), similar studies were less reported in the interspecies relation between cyanobacteria and green alga. Different ecological roles have been attributed to the production of the allelochemicals by cyanobacteria, including phytoplankton succession, bloom formation, resource and interference competition and invasive fitness (Figueredo et al. 2007). However, the influence of the allelochemicals by other species on cyanobacteria has been overlooked in the past study. Our study may provide a possible method for cyanobacterial controlling. For one thing, we could use the green alga-condition media but not the green alga directly to inhibit $M$. aeruginosa growth. For another, although cyanobacteria are harmful for the environment, the condition media from cyanobacteria also could be viewed as the resource and used to stimulate the growth of S. obliquus, which is advantaged for gaining more green alga-condition media and food for fish. Clearly, we foresee a future in the additional experimental work as follows: (1) identify and isolate the chemical substances with allelopathic effect in the conditioned media, (2) produce several cyanobacterial inhibitors based on these substances. (3) point out a new environmental friendly biological control measures by three steps: cyanobacterial inhibition by green alga, while green alga growth stimulation by cyanobacteria and green alga grazing by fish.

\section{Conclusion}

In this study, we used the common freshwater green alga $S$. obliquus as the candidate cyanobacteria-control species by its allelopathic effect. The population growth of the cyanobacteria $M$. aeruginosa was inhibited significantly by the green alga-conditioned media while there was a strong and significant positive effect of culture media from $M$. aeruginosa on the growth of green alga. Our results are useful and valuable for producing new possible cyanobacterial inhibitors and applying a new environmental friendly biological control method in future as the experimental evidence.

Acknowledgments This work was supported by National Special Purpose on Public Welfare of Environmental Protection Foundation (200809016), Jiangsu Key Lab of Environmental Engineering Open Foundation (KF2012008).

\section{References}

Adolf JA, Krupatkina D, Bachvaroff T, Place AR (2007) Karlotoxin mediates grazing by Oxyrrhis marina on strains of Karlodinium veneficum. Harmful Algae 6:400-412

Barrett PRF, Curnow JC, Littlejohn JW (1996) The control of diatom and cyanobacterial blooms in reservoirs using barley straw. Hydrobiologia 340:307-311

Cohen JH, Tester PA, Forward RB (2007) Sublethal effects of the toxic dinoflagellate Karenia brevis on marine copepod behavior. J Plankton Res 29:301-315

Collins M (1978) Algal toxins. Microbiol Rev 42:725-746

Figueredo CC, Giani A, Bird DF (2007) Does allelopathy contribute to Cylindrospermopsis raciborskii (cyanobacteria) bloom occurrence and geographic expansion? J Phycol 43:256-265

Fistarol GO, Legrand C, Graneli E (2003) Allelopathic effect of Prymnesium parvum on a natural plankton community. Mar Ecol Prog Ser 255:115-125

Gross EM (2003) Allelopathy of aquatic autotrophs. Crit Rev Plant Sci 22:313-339

Guo R, Snell TW, Yang J (2011) Ecological strategy of rotifer (Brachionus calyciflorus) exposed to predator- and competitorconditioned media. Hydrobiologia 658:163-171

Hay ME (2009) Marine chemical ecology: chemical signals and cues structure marine populations, communities, and ecosystems. Annu Rev Mar Sci 1:193-212

Hullebusch EV, Deluchat V, Chazal PM, Baudu M (2002) Environmental impact of two successive chemical treatments in a small shallow eutrophied lake: part I case of aluminium sulphate. Environ. Pollut 120:617-626

Jonsson PR, Pavia H, Toth G (2009) Formation of harmful algal blooms cannot be explained by allelopathic interactions. Proc Natl Acad Sci USA 106:11177-11182

Leãoa PN, Pereirab AR, Liuc WT, Ngd J, Pevznerd PA, Dorresteinb PC, Königf GM, Vasconcelosa VM, Gerwickb WH (2010) Synergistic allelochemicals from a freshwater cyanobacterium. Proc Natl Acad Sci USA 107:11183-11188

Legrand C, Rengefors K, Fistarol GO (2003) Allelopathy in phytoplankton-biochemical, ecological and evolutionary aspects. Phycologia 42:406-419

Lurling M (2003) The effect of substances from different zooplankton species and fish on the induction of defensive morphology in the green alga Scenedesmus obliquus. J Plankton Res 25:979-989

Mazzillo FFM, Ryan JP, Silver MW (2011) Parasitism as a biological control agent of dinoflagellate blooms in the California Current System. Harmful Algae 10:763-773

Mehner T, Arlinghaus R, Berg S, Dorner H, Jacobsen L, Kasprzak P, Koschel R, Schulze T, Skov C, Wolter C, Wysujack K (2004) How to link biomanipulation and sustainable fisheries management: a step-by-step guideline for lakes of the European temperate zone. Fisheries Manag Ecol 11:261-275

Mulderij G, Mooij WM, Donk EV (2005) Allelopathic growth inhibition and colony formation of the green alga Scenedesmus obliquus by the aquatic macrophyte Stratiotes aloides. Aquat Ecol 39:11-21

Paerl HW, Fulton RS, Moisander PH, Dyble J (2001) Harmful freshwater algal blooms, with an emphasis on cyanobacteria. Sci World J 1:76-113

Wu Y, Kerr PG, Hu Z, Yang L (2010) Removal of cyanobacterial bloom from a biopond-wetland system and the associated response of zoobenthic diversity. Bioresour Technol 101:3903-3908

Zhang X, Warming TP, Hu HY, Christoffersen KS (2009) Life history responses of Daphnia magna feeding on toxic Microcystis aeruginosa alone and mixed with a mixotrophic Poterioochromonas species. Water Res 43:5053-5062 